\title{
Spatial interactions between subsurface bacterial colonies in a model system: a territory model describing the inhibition of Listeria monocytogenes by a nisin-producing lactic acid bacterium
}

I School of Pure and Applied Biology, University of Wales Cardiff, Cardiff CF1 3TL, UK

2 Institute of Food Research, Norwich Research Park, Colney, Norwich NR4 7UA, UK

\author{
Linda V. Thomas, ${ }^{1}$ Julian W. T. Wimpenny ${ }^{1}$ and Gary C. Barker ${ }^{2}$
}

Author for correspondence: Linda V. Thomas. Tel: +44 1222874000 ext. 6806. Fax: +44 1222874305 . e-mail: sablvt@cf.ac.uk

The effect of spatial separation on interactions between subsurface bacterial colonies was tested using a model system: the inhibition of Listeria monocytogenes by nisin-producing and nisin-non-producing Lactococcus lactis subsp. lactis. Separation distance was controlled by altering the number of inoculum organisms within the agar. Mean separation distance was calculated by determining the mean volume available to each cell at the start of the experiment. Inhibition was assessed by comparing the growth of L. monocytogenes in pure culture with its growth in the presence of Lac. lactis subsp. lactis. Increasing the distance between colonies resulted in an exponential decrease in inhibition. When $L$. monocytogenes and Lac. lactis subsp. lactis colonies were within $100 \mu \mathrm{m}$ of each other, the increase in cell numbers per $\boldsymbol{L}$. monocytogenes colony was only 0.6 c.f.u. (which indicated some cells had become non-viable). This was a log reduction of 3.5 compared to the pure culture control. A separation distance of $1000 \mu \mathrm{m}$ resulted in a L. monocytogenes colony growth increment of $2.5 \times 10^{2}$ c.f.u. per colony, a log reduction of 3.0 compared to the control. Increasing the separation distance to $3000 \mu \mathrm{m}$ resulted in a $L$. monocytogenes colony growth increment of $1.3 \times 10^{6}$ c.f.u. per colony, a log reduction of 0.9 compared to the control. The effects of nisin and acidity were investigated by using a nisin-non-producing strain of Lac. lactis subsp. lactis and by buffering the medium. Data were obtained for the effect of separation on inhibition, as well as competition between colonies of the same species. The inhibition was mathematically described in terms of a simplified 'territory' model of immobilized bacterial growth. There was a strong qualitative agreement between the mathematical model and the experimental data. It was concluded that the phenomenon of propinquity is of important consideration when modelling and predicting microbial growth within solid food systems.

Keywords: Listeria monocytogenes, propinquity, nisin, lactic acid bacteria, territory model, spatial separation

\section{INTRODUCTION}

Lactic acid bacteria (LAB) have been used in food for centuries both to preserve and enhance the flavour of the food. LAB are 'generally recognized as safe' (GRAS)

Abbreviation: LAB, lactic acid bacteria. and can be described as 'biopreservatives', a natural method of food preservation. LAB inhibit the growth of other organisms by competing for nutrients and by producing metabolic products such as bacteriocins, organic acids, ammonia and hydrogen peroxide (Schillinger et al., 1996; Vandenbergh, 1993). The LAB Lactococcus lactis subsp. lactis produces the bacteriocin nisin, which is widely used in food. Nisin is a small 
polypeptide which inhibits the vegetative growth of Gram-positive organisms and prevents the outgrowth of endospores (Hurst, 1981; Delves-Broughton, 1990).

$\mathrm{LAB}$ are used as starter cultures in fermented meat and dairy products, many of which are of solid or semi-solid consistency (Adams \& Moss, 1995). In solid food systems organisms are immobilized and grow as colonies at fixed positions within the food matrix. In such systems the spatial separation, or propinquity, of the colonies is a variable which can influence the outcome of potential interactions between them (Wimpenny et al., 1995). In this paper we use a simple method to demonstrate the effect of distance on interactions between separated and immobilized colonies of Lac. lactis subsp. lactis (nisinproducing and -non-producing) and Listeria monocytogenes growing in agar. The distance effect is described mathematically in terms of a territory model. Data obtained from the propinquity experiments are compared with this model.

\section{METHODS}

Organisms and media. Nisin-producing and -non-producing Lac. lactis subsp. lactis strains were used: strain FI5876 (nisinproducing) and strain MG1614 (nisin-non-producing) were supplied by the Institute of Food Research, Norwich. L. monocytogenes F4642 was supplied by Campden Food and Drink Research Association. The strains were grown on BHIYEGA medium containing $\left(\mathrm{g} \mathrm{l}^{-1}\right)$ : brain heart infusion (Difco), 37; yeast extract (Difco), 3; glucose (BDH), 3; and agar (Difco), 8. Broth cultures were grown in the same medium without the addition of agar (BHIYEGB). To neutralize the acid produced by the lactococci, $3 \mathrm{~g} \mathrm{CaCO}_{3} \mathrm{I}^{-1}$ was added to the agar. The $\mathrm{CaCO}_{3}$ was well suspended to ensure even dispersion within the agar once it had set (Costilow, 1981). Purified nisin (Aplin \& Barrett) was added to certain control plates. The organisms were maintained on glass beads at $-70^{\circ} \mathrm{C}$ (Jones et al., 1984).

Propinquity experiments. Strains were plated onto BHIYEGA, grown at $30^{\circ} \mathrm{C}$ overnight and inoculated into liquid medium. Liquid cultures were grown to exponential phase for about $6 \mathrm{~h}$ at $30^{\circ} \mathrm{C}$, adjusted to approximately equal turbidity and serially diluted by factors of ten. Aliquots of each dilution were used to inoculate pour-plates. The inhibitory effect of nisin together with acidity on L. monocytogenes was examined by growing the organisms in BHIYEGA with no added $\mathrm{CaCO}_{3}$. The inhibitory effect of nisin alone was investigated by growing the organisms in BHIYEGA containing $\mathrm{CaCO}_{3}$. To distinguish inhibition effects due to nisin and acidity, the growth of L. monocytogenes in the presence of the nisin-producing and -nonproducing strains of Lac. lactis subsp. lactis was compared. The following sets of plates were prepared (in triplicate): pure cultures of (i) L. monocytogenes, (ii) Lac. lactis subsp. lactis FI5876 (nisin-producing) and (iii) Lac. lactis subsp. lactis MG1614 (nisin-non-producing); and mixed cultures of (iv) $L$. monocytogenes and Lac. lactis subsp. lactis F15876 (nisinproducing) and (v) L. monocytogenes and Lac. lactis subsp. lactis MG1614 (nisin-non-producing). Mixed cultures were inoculated from the dilution series of both organisms.

The following additional control plates were prepared to check that nisin activity was not affected by $\mathrm{CaCO}_{3}$ : (i) $L$. monocytogenes in BHIYEGA with $100 \mathrm{IU}$ nisin $\mathrm{ml}^{-1}$ and (ii) $L$. monocytogenes in BHIYEGA with $100 \mathrm{IU}$ nisin $\mathrm{ml}^{-1}$ and $3 \mathrm{~g}$
$\mathrm{CaCO}_{3} \mathrm{l}^{-1}$. Further control plates were prepared in each set of experiments to check that inhibition due to the production of nisin and acidity occurred in BHIYEGA with no additions.

Immediately after inoculation, when the solid media had set, the number of viable cells inoculated into each plate was determined by taking samples for viable counts (as described below). The plates were then incubated at $30^{\circ} \mathrm{C}$ for $18 \mathrm{~h}$. In pour-plates inoculated with low numbers of cells, the inoculation count was also estimated by directly counting developing colonies in the plates. The colonies were seen to be evenly distributed and well separated. Viable counts were used to measure growth after $18 \mathrm{~h}$. Inhibition was assessed by comparing the growth achieved in pure culture with the growth of the same strain in mixed culture.

Viable counts. Three $23 \mathrm{~mm}$ diameter agar plugs were removed from each $90 \mathrm{~mm}$ diameter plate (which were then incubated for $18 \mathrm{~h}$ ), placed into $2.5 \mathrm{ml}$ Ringers solution and homogenized using a hand-held homogenizer. Aliquots of the resulting suspension were serially diluted and plated onto selective media. All counts were performed in triplicate and results were calculated as $\log$ c.f.u. $\mathrm{ml}^{-1} \pm \mathrm{SD}$, unless otherwise stated. The growth increment of the colonies within the same plates after $18 \mathrm{~h}$ was expressed as the mean increase in total number of viable cells per colony, estimated by dividing the count obtained after incubation by the count at inoculation. This value was termed the 'colony growth increment' and was expressed as c.f.u. per colony (see Figs 1-3).

The Lac. lactis subsp. lactis strains were selectively counted from the competition and pure control plates by growth at $30^{\circ} \mathrm{C}$ overnight on MRS agar (Oxoid). L. monocytogenes was isolated by growth at $30^{\circ} \mathrm{C}$ overnight on Oxford Listeria Selective Agar (Merck).

\section{RESULTS}

\section{Estimation of separation distance between colonies}

The mean distance between colonies was determined by the initial concentration of cells, $n(0) \mathrm{cm}^{-3}$. On average each colony occupied a volume $1 / n(0) \mathrm{cm}^{3}$ and if we approximate this volume as a sphere with radius $r$, this gives :

$(4 / 3) \pi r^{3}=1 / n(0)$

The mean colony separation is twice the sphere radius:

$2 \sqrt{\frac{3}{4 \pi n(0)}} \mathrm{cm}$, or $2 \sqrt[3]{\frac{3 \times 10^{12}}{4 \pi n(0)}} \mu \mathrm{m}$

For mixed cultures $n(0)$ is the sum of the inoculation densities for L. monocytogenes and Lac. lactis subsp. lactis. In practice, in a well mixed system the colonies have a distribution of separations with Poisson statistics - some colonies are closer than the mean separation and others are much farther apart. For this first model of nisin inhibition all colony separations were considered to be equal to the mean separation.

Inhibition was assessed in each case by comparison of the colony growth increment in mixed culture with that of the pure culture control, and was expressed by calculating the log reduction (subtracting the log of the colony growth increment in mixed culture from that achieved in pure culture). 


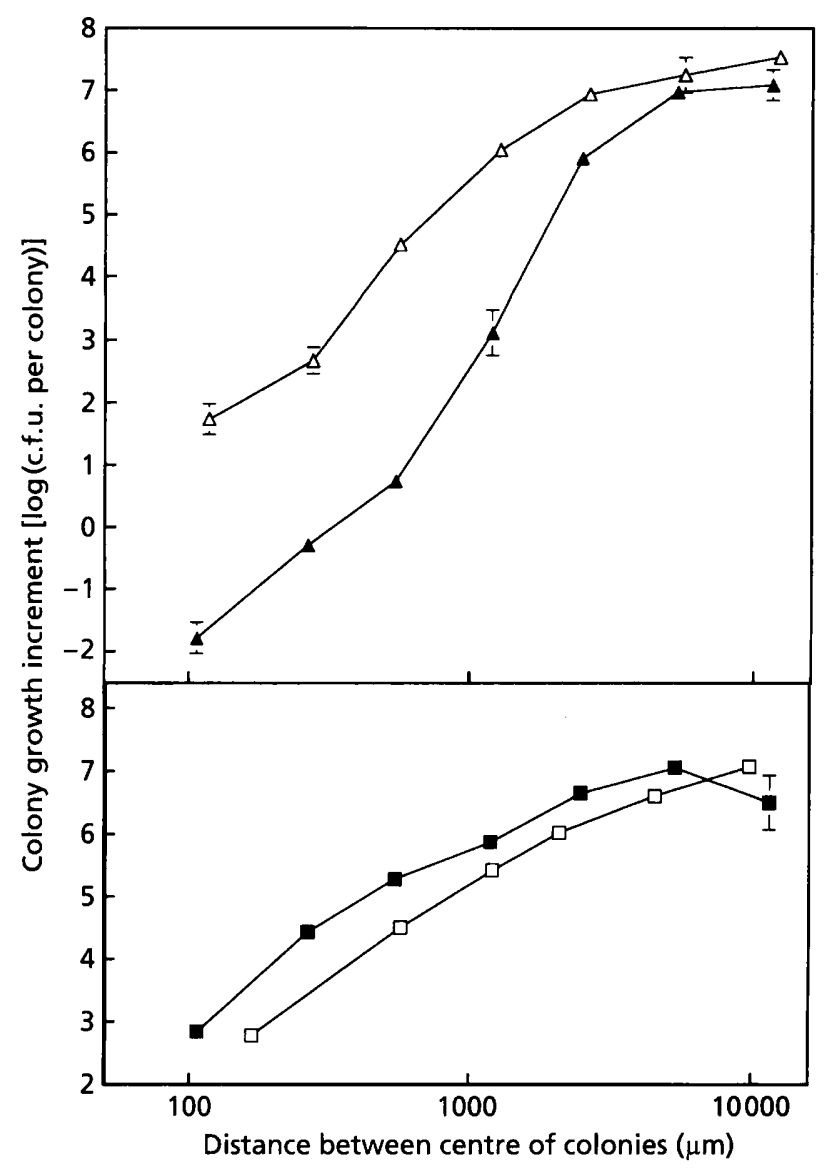

Fig. 1. Effect of spatial separation on inhibition of $L$. monocytogenes by the nisin-producing Lac. lactis subsp. lactis FI5876 in unbuffered medium. The growth increment of subsurface colonies incubated in BHIYEGA for $18 \mathrm{~h}$ at $30^{\circ} \mathrm{C}$ was measured for $L$. monocytogenes in pure culture $(\triangle)$ and in mixed culture with Lac. lactis subsp. lactis FI5876 (A), and for Lac. lactis subsp. lactis FI5876 in pure culture $(\square)$ and in mixed culture with $L$. monocytogenes ( $\mathbf{D})$.

\section{Effect of spatial separation on inhibition of $L$. monocytogenes due to the growth of nisin-producing Lac. lactis subsp. lactis FI5876 in unbuffered medium}

The effect of inhibition due to the combined effects of acidity, nisin and other possible interacting effects (such as other metabolic products, substrate, etc.) was demonstrated by growing the nisin-producing Lac. lactis subsp. lactis strain FI5876 in unbuffered BHIYEGA medium (with no added $\mathrm{CaCO}_{3}$ ). The undiluted inocula were (mean $\log$ c.f.u. $\mathrm{ml}^{-1} \pm \mathrm{SD}$ ): L. monocytogenes, $6 \cdot 07 \pm 0 \cdot 22 ;$ Lac. lactis subsp. lactis, FI5876, 5.61 $\pm 0 \cdot 20$.

At a mean distance of about $110 \mu \mathrm{m}$ the L. monocytogenes colony growth increment in the presence of the nisin-producing lactococcal strain was $6.1 \times 10^{-1}$ c.f.u. per colony, a log reduction of $3 \cdot 5$ compared to the pure culture control (Fig. 1). This indicated that many of the L. monocytogenes cells had become non-viable during incubation in the presence of the lactococcal strain. The degree of inhibition of L. monocytogenes in

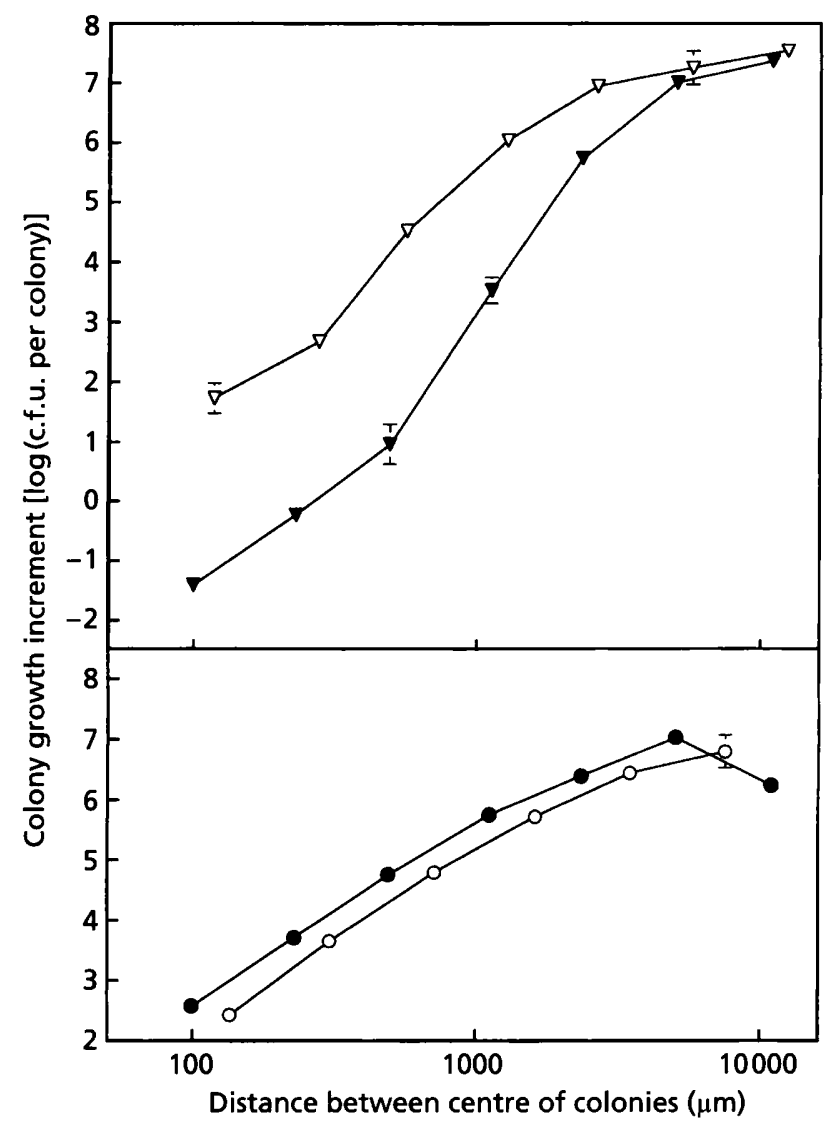

Fig. 2. Effect of spatial separation on inhibition of $L$. monocytogenes by the nisin-non-producing Lac. lactis subsp. lactis MG1614 in unbuffered medium. The growth increment of subsurface colonies incubated in BHIYEGA for $18 \mathrm{~h}$ at $30^{\circ} \mathrm{C}$ was measured for $L$. monocytogenes in pure culture $(\nabla)$ and in mixed culture with Lac. lactis subsp. lactis MG1614 ( $\nabla)$ and for Lac. lactis subsp. lactis MG1614 in pure culture $(O)$ and in mixed culture with $L$. monocytogenes (O).

the mixed culture fell exponentially as a function of increasing colony separation. At a separation distance of about $1200 \mu \mathrm{m}$ the colony growth increment of $L$. monocytogenes in the presence of the lactococcal strain was $1.3 \times 10^{3}$ c.f.u. per colony, a log reduction of 2.8 compared to the pure control. At $5000 \mu \mathrm{m}$ separation the colony growth increment of $L$. monocytogenes in the presence of the lactococcal strain increased to $9 \times 10^{6}$ c.f.u. per colony, a log reduction of $0 \cdot 4$ compared to the pure control (Fig. 1). Growth of the lactococcal strain was not inhibited by L. monocytogenes and in fact showed a slight increase in c.f.u. per colony. However, if the separation distance between lactococcal cells was used (instead of the distance between any two colonies, either L. monocytogenes or Lac. lactis subsp. lactis), the pure and mixed culture plots in Fig. 1 were very similar. Pure cultures of both species demonstrated the recognized 'crowding' effect caused by competition for essential nutrients. The maximum colony size for the pure cultures was about $10^{7}$ c.f.u. per colony where the separation distance was greater than about $3000 \mu \mathrm{m}$ (Fig. 1). 


\section{Effect of spatial separation on inhibition of L. monocytogenes due to the growth of nisin- non-producing Lac. lactis subsp. lactis MG1614 in unbuffered medium}

The effect of inhibition due to the production of acid and other possible interactive effects but not due to nisin, was demonstrated by growing $L$. monocytogenes with the nisin-non-producing Lac. lactis subsp. lactis MG1614 in unbuffered BHIYEGA medium (with no added $\mathrm{CaCO}_{3}$ ). Inhibition was assessed as above. The undiluted inocula were (mean log c.f.u. $\mathrm{ml}^{-1} \pm \mathrm{SD}$ ): $L$. monocytogenes, $6 \cdot 07 \pm 0 \cdot 22$; Lac. lactis subsp. lactis MG1614, 5·89 $\pm 0 \cdot 12$.

At close separation distances the growth of the $L$. monocytogenes colonies was inhibited by the lactococcal strain. At a mean separation distance of about $100 \mu \mathrm{m}$ the colony growth increment of L. monocytogenes in the presence of the lactococcal strain was $2.6 \times 10^{-1}$ c.f.u. per colony, a $\log$ reduction of $3 \cdot 1$ compared to the pure control (Fig. 2). Increasing the colony separation distance decreased L. monocytogenes colony inhibition as before (see above). At a separation distance of $1100 \mu \mathrm{m}$ the colony growth increment of $L$. monocytogenes in mixed culture was $3 \times 10^{3}$ c.f.u. per colony, a log reduction of $2 \cdot 2$ compared to the pure control. At $5000 \mu \mathrm{m}$ the colony growth increment of $L$. monocytogenes in mixed culture was $1 \times 10^{7}$ c.f.u. per colony, a log reduction of 0.2 compared to the pure control. The Lac. lactis subsp. lactis MG1614 colonies and pure culture controls were similar to those described above (Fig. 2).

\section{Effect of spatial separation on inhibition of L. monocytogenes due to nisin-producing Lac. lactis subsp. lactis F15876 in buffered medium}

The effect of inhibition by nisin and other possible interactive effects, but not due to acidity, was demonstrated by growing $L$. monocytogenes with nisinproducing Lac. lactis subsp. lactis FI5876 in BHIYEGA medium containing $\mathrm{CaCO}_{3}$, which effectively neutralized the acid produced by the lactococcal strain (Jones et al., 1984). The undiluted inocula were (mean $\log$ c.f.u. $\mathrm{ml}^{-1} \pm \mathrm{SD}$ ): L. monocytogenes, $4 \cdot 74 \pm 0 \cdot 06$; Lac. lactis subsp. lactis FI5876, $5 \cdot 23 \pm 0 \cdot 11$; Lac. lactis subsp. lactis MG1614, 5·47 $\pm 0 \cdot 10$.

In this medium the nisin-non-producing Lac. lactis subsp. lactis MG1614 caused only minor inhibition of $L$. monocytogenes in mixed culture, even when colonies were within $1000 \mu \mathrm{m}$ of each other (Fig. 3). This small inhibition was not due to nisin or acidity. In contrast, the nisin-producing strain Lac. lactis subsp. lactis FI5876 reduced the number of $L$. monocytogenes colonies by over $10^{4}$ c.f.u. per colony when colonies were within about $200 \mu \mathrm{m}$ of each other. When the colonies were $940 \mu \mathrm{m}$ apart the colony growth increment was $2 \times 10^{2}$ c.f.u. per colony, a log reduction of $3 \cdot 2$ compared to the pure control, but at a separation distance of $4800 \mu \mathrm{m}$ the colony increment was $1 \times 10^{6}$ c.f.u. per colony, a log reduction of only 0.6 compared to the pure control.

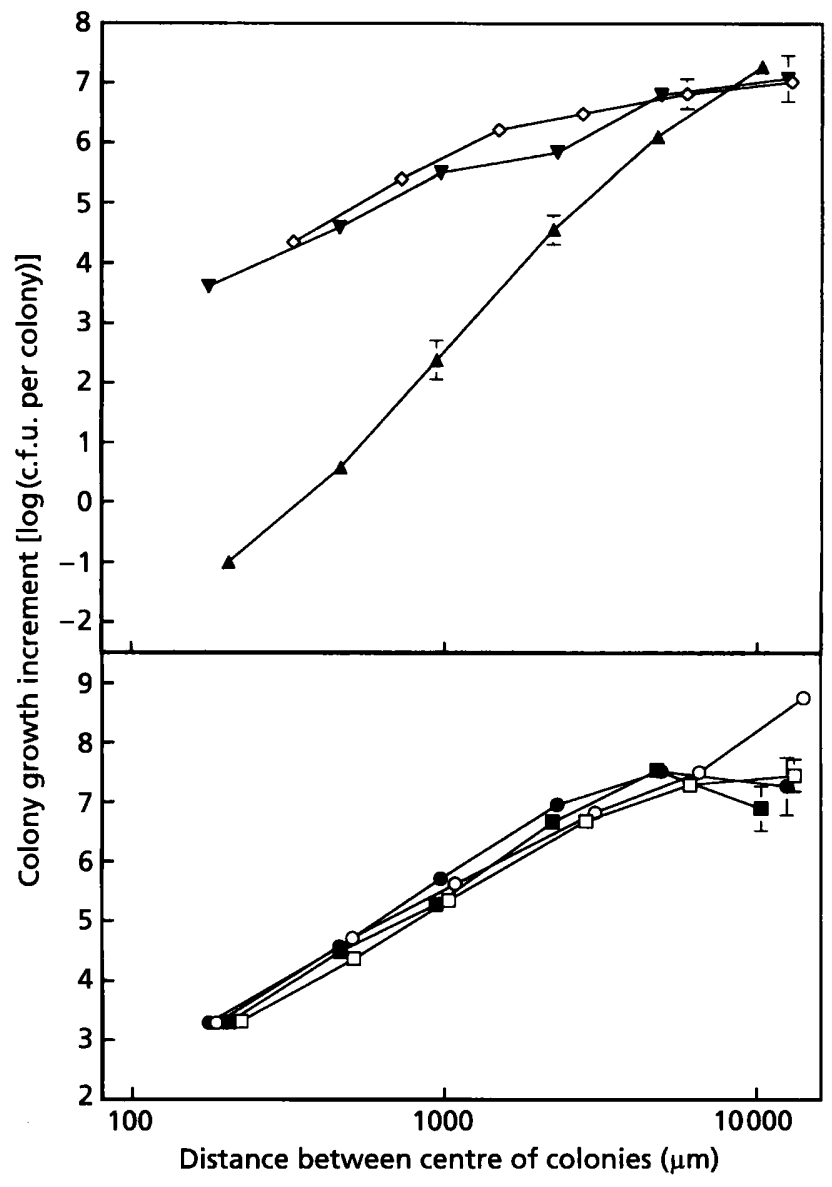

Fig. 3. Effect of spatial separation on inhibition of $L$. monocytogenes by the nisin-producing Lac. lactis subsp. lactis FI5876 in buffered medium. The growth increment of subsurface colonies incubated in BHIYEGA containing $\mathrm{CaCO}_{3}$ for $18 \mathrm{~h}$ at $30^{\circ} \mathrm{C}$ was measured for $L$. monocytogenes in pure culture $(\diamond)$, in mixed culture with Lac. lactis subsp. lactis FI5876 (nisin-producing) (A) and in mixed culture with Lac. lactis subsp. lactis MG1614 (nisin-non-producing) ( $\nabla)$, for Lac. lactis subsp. lactis FI5876 in pure culture ( $\square$ ) and in mixed culture with L. monocytogenes ( $\square$ ), and for Lac. lactis subsp. lactis MG1614 in pure culture $(O)$ and in mixed culture with $L$. monocytogenes (O).

Growth of lactococcal colonies was not inhibited by L. monocytogenes but showed the inhibitive effect of competitive interactions between its own colonies, which decreased with increasing separation distance (Fig. 3). In control plates containing pure nisin, the nisin activity (as measured by L. monocytogenes inhibition) was not affected by the $\mathrm{CaCO}_{3}$ added to the medium.

\section{A 'territory' model}

The inhibition of L. monocytogenes colonies by nisinproducing Lac. lactis subsp. lactis FI5876 can be understood in terms of a territory model of immobilized bacterial growth. In a territory model the substrate is geometrically partitioned according to the initial distribution of bacterial growth sites, and each colony is supported exclusively by its local environment. Thus, a 
cell at the centre of a large volume of otherwise unoccupied substrate has a potential for more growth than a similar cell in an environment crowded by other growth sites. In a first approximation we assume that the growth at each site is exponential:

$N_{i}(t)=10^{t / t_{10}}$

until either the local substrate is exhausted or the growth process is interrupted for a different reason. Here $N_{\mathrm{i}}(t)$ is the number of cells at the $i$ th growth site at time $t$ and $t_{10}$ is the decimal increment time. When we consider the inhibition process, exponential growth of L. monocytogenes colonies is terminated by an increase in the local concentration of nisin which, in turn, is caused by the growth of nearby colonies of nisinproducing Lac. lactis subsp. lactis FI5876.

Territory models may be used to account for many complex transitions in population dynamics that are caused by environmental changes. Here we will only consider L. monocytogenes colonies that cease growing when they are triggered by a nearby colony that produces nisin. The time at which the growth of a $L$. monocytogenes colony would be terminated by a "nisin switch' is determined by the distance between the colony and the nearest neighbouring colony of the lactococcal strain and by the rate at which the nisin signal is transported through the medium. Clearly the colony separations depend on the initial concentration and distribution of growth sites in the mixed inoculation and this will be included in the model below. In addition we will assume that the transport of nisin through the growth medium is purely diffusive so that during a time interval $t$ the signal will propagate a distance $\sqrt{(6 D t)}$ where $D$ is the diffusion coefficient of nisin. This approximation, which is a good one in situations where diffusion is the fastest inhibitor transport mechanism and the inhibition switch does not have a threshold, omits the role played by a variable inhibitor production rate. Whilst the details of the transport and switching processes have not been included explicitly in this model, the important point is that the nisin signal gets progressively slower as it propagates away from its source and this is in contrast to the exponentially increasing rate at which a growing colony exhausts its local supply of substrate.

This scheme together with stoichiometric details for the consumption of substrate and statistical distributions of territory volumes and of colony separations is sufficient to specify the growth of a mixed population of immobilized, interacting bacteria. Here, for simplicity, we will consider only the 'uniform' territory model in which all the territories have the same volume which is equal to the reciprocal of the inoculation density. This approximation is not essential but it proves satisfactory for the discussion of nisin inhibition in the experimental configuration explored above. Thus, in the uniform territory model, for a pure culture of L. monocytogenes we may express the population growth as:

$\begin{aligned} n(t) & =n(0) 10^{t / t_{10}} & & t<t_{\text {sat }} \\ & =n_{\infty} & & t \geqslant t_{\text {sat }}\end{aligned}$

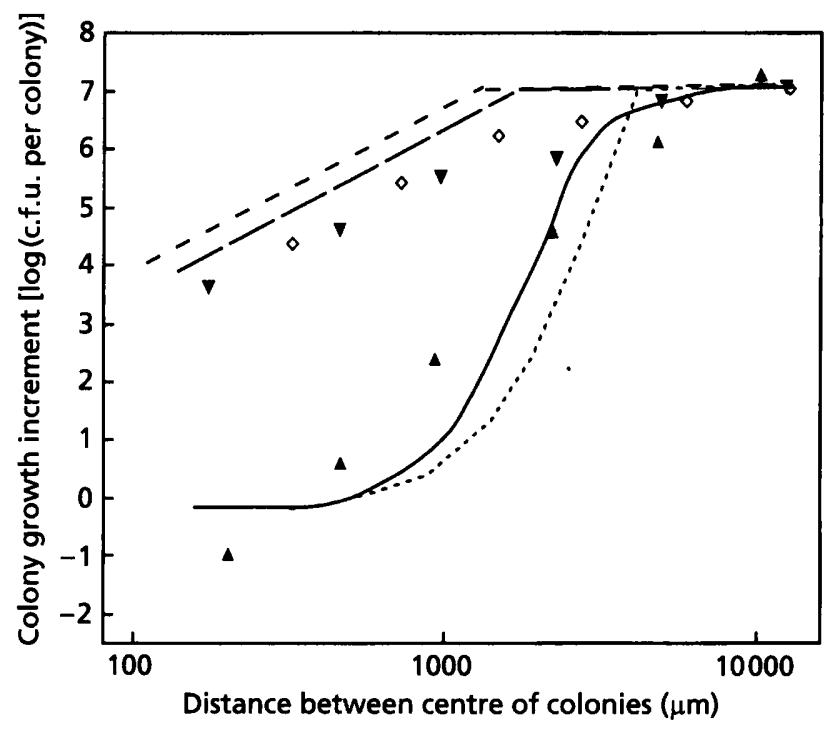

Fig. 4. A territory model showing the effect of spatial separation of immobilized cells on the colony growth increment of $L$. monocytogenes. Inhibition of $L$. monocytogenes by nisin-producing Lac. lactis subsp. lactis F15876 in a buffered medium. Short-dashed line, predicted colony growth increments of $L$. monocytogenes in pure culture; long-dashed line, predicted colony growth increment of $L$. monocytogenes in mixed culture with nisin-non-producing Lac. lactis subsp. lactis MG1614; dotted and solid lines, predicted colony growth increment of $L$. monocytogenes in mixed culture with nisinproducing Lac. lactis subsp. lactis FI5876 using a uniform territory model (dotted line; equation 11) and using a more realistic distribution of colony separations (solid line). Data from Fig. 3 are superimposed (see Fig. 3 legend for details).

where $t_{\text {sat }}=t_{10}\left[\log n_{\infty}-\log (n(0))\right]$ is the time that each colony takes to exhaust its local environment of substrate, $n(0)$ is the inoculation density and $n_{\infty}$ is the density of cells that results from the full conversion of the substrate. In this simple case $n_{\infty}$ is a full expression of the stoichiometry of the growth. From above, the colony increment after a fixed period of inoculation, $t_{\mathbf{x}}$, may be expressed as:

$$
\begin{array}{rlrl}
I & =\log \left[\frac{n\left(t_{\mathrm{x}}\right)}{n(0)}\right]=t_{\mathrm{x}} / t_{10} & & t_{\mathrm{x}}<t_{\text {sat }} \\
& =\log n_{\infty}-\log [n(0)] & t_{\mathrm{x}} \geqslant t_{\text {sat }}
\end{array}
$$

In turn the reciprocal cube root of the inoculation density is proportional to the mean distance between colonies, $\langle r\rangle$, so that by substituting $n(0)=3 / 4 \pi r^{3}$ and $\langle r\rangle \sim 2 r$ into the above we obtain:

$$
\begin{array}{rlrl}
I & =t_{\mathrm{x}} / t_{10} & & \log \langle r\rangle>\left(t_{\mathrm{x}} / t_{10}-\log n_{\infty}+c\right) / 3 \\
& =c+\log n_{\infty}+3 \log \langle r\rangle & \log \langle r\rangle \leqslant\left(t_{\mathrm{x}} / t_{10}-\log n_{\infty}+c\right) / 3
\end{array}
$$

where $c$ is a small constant, such as $\log (4 / 3 \pi)$, that depends on the local geometry of a colony. This expression can be represented by two straight line segments in a plot of $I$ against $\log \langle r\rangle$; this is shown by the short-dashed line in Fig. 4. For comparison with the experimental data in Fig. 4, we have used $c=0$, which 
does not lead to serious errors, and $n_{\infty}=10^{9}$ as an estimate of the maximum concentration of bacteria. The growth of $L$. monocytogenes in uninhibited environments indicates that a population increases by approximately seven orders of magnitude during the incubation period so that we have used $t_{\mathrm{x}} / t_{10}=7$. The constant segment corresponds to colonies that are still in exponential growth at the incubation time and the sloping segment, which has a gradient 3 , represents colonies that have been limited by the available substrate prior to the full incubation.

In the absence of a specific inhibition effect, the introduction of a second species of bacteria, such as the nisin-non-producing Lac. lactis subsp. lactis MG1614, only has a small impact on the population dynamics for L. monocytogenes. The changes arise from the competition between the two populations for the same substrate so that, in the presence of the lactococcal strain, the maximum value of $L$. monocytogenes density, $n(\infty)$, is less than the maximum density of L. monocytogenes in pure culture, $n_{\infty}$. Again for simplicity and to parallel the experiments described above, we consider a limited version of the territory model, in which both species of bacteria have identical kinetics, i.e. $t_{10}(L$. monocytogenes $)=t_{10}$ (Lac. lactis subsp. lactis), and in which the two inoculation densities are equal so that $n(\infty)=\left(n_{\infty}\right) / 2$. Then, as before, the expression for the colony increment of L. monocytogenes is:

$$
\begin{array}{rlrl}
I & =t_{\mathrm{x}} / t_{10} & & \log \langle r\rangle>\left(t_{\mathrm{x}} / t_{10}-\log n_{\infty}+\log 2\right) / 3 \\
& =\log n_{\infty}+3 \log \langle r\rangle & \log \langle r\rangle \leqslant\left(t_{\mathrm{x}} / t_{10}-\log n_{\infty}+\log 2\right) / 3
\end{array}
$$

where a factor $\log 2$ in the expression for the ranges has arisen from the symmetry assumptions above. This behaviour, shown as the long-dashed line in Fig. 4, is a simple shift of the pure culture response. This shift represents the onset of substrate exhaustion at larger $L$. monocytogenes $-L$. monocytogenes separations caused by the lactococcal colonies using half of the available substrate.

In the presence of nisin-producing Lac. lactis subsp. lactis FI5876, the growth of L. monocytogenes is more complex because the exponential regime of colony expansion may be terminated, by nisin inhibition, prior to the exhaustion of the local substrate. This occurs if the colony of $L$. monocytogenes is sufficiently close to a site of lactococcal growth. Thus at time $t$, the number of cells in the $i$ th colony of $L$. monocytogenes, $N_{\mathrm{i}}(t)$, may be written as:

$$
\begin{aligned}
N_{\mathrm{i}}(t) & =10^{t / t_{10}} & & t<t_{\mathrm{sat}}, t_{\mathrm{i}}^{\mathrm{N}} \\
& =10^{t_{\mathrm{i}}^{\mathrm{N}} / t_{10}} & & t>t_{\mathrm{i}}^{\mathrm{N}} \text { and } t_{\mathrm{i}}^{\mathrm{N}}<t_{\text {sat }} \\
& =10^{t_{\mathrm{sat}} / t_{10}} & & t>t_{\text {sat }} \text { and } t_{\text {sat }}<t_{\mathrm{i}}^{\mathrm{N}}
\end{aligned}
$$

where $t_{\mathbf{i}}^{\mathbf{N}}$ is the time until inhibition by nisin for the $i$ th L. monocytogenes colony. To evaluate these inhibition times, here we simply consider the nearest $L$. monocytogenes-Lac. lactis subsp. lactis distance to be equal to a constant for all L. monocytogenes colonies; this constant is proportional to the reciprocal of the cube root of the Lac. lactis subsp. lactis inoculation density. A more realistic situation, in which these separations have a well-defined statistical distribution, such as the Poisson distribution, may be easily incorporated into this analysis and leads to integral expressions for the population growth and the colony increments given below. Then, since in the symmetric model the inoculation densities for L. monocytogenes and Lac. lactis subsp. lactis are identical, we may express the time to inhibition for $L$. monocytogenes colonies as:

$t^{\mathrm{N}} \propto n(0)^{-2 / 3} / 6 D$

where we have used the square root relationship between distances travelled and elapsed time for the diffusing nisin. In terms of the mean separation of L. monocytogenes colonies:

$t^{\mathrm{N}}=\left(\mathrm{k}\langle r\rangle^{2}\right) / 6 D$

where $\mathrm{k}$ is a numerical constant, of order unity, that depends on local geometry. By combining equations (8) and (10) we can express the colony increment for L. monocytogenes in symmetric mixed populations with nisin-producing Lac. lactis subsp. lactis FI5876 as:

$$
\begin{array}{rlrl}
I & =t_{\mathrm{x}} / t_{10} & & \log \langle r\rangle>(1 / 2) \log \left(6 D t_{\mathrm{x}} / \mathrm{k}\right) \\
& =\left(k\langle r\rangle^{2}\right) /\left(6 D t_{10}\right) & \log \langle r\rangle \leqslant(1 / 2) \log \left(6 D t_{\mathrm{x}} / \mathrm{k}\right) \\
& =\log n_{\infty}+3 \log \langle r\rangle & \log \langle r\rangle \leqslant\left(t_{\mathrm{x}} / t_{10}-\log n_{\infty}+\log 2\right) / 3
\end{array}
$$

The behaviour described by equation (11) is shown as a dotted line in Fig. 4. We have used $D / \mathrm{k}=10^{-6} \mathrm{~cm}^{2} \mathrm{~s}^{-1}$ so that the inhibitor has a diffusion coefficient typical for polypeptide-like molecules in water. Many features of the behaviour, particularly at large separations, coincide with those for uninhibited colonies. However, at intermediate and small separations, there is a rapid change in colony increment, quadratic in $\langle r\rangle$ (exponential in $\log \langle r\rangle)$, that corresponds to cultures that have $L$. monocytogenes-Lac. lactis subsp. lactis separations that are traversed by a nisin signal before a colony can exhaust its local supply of substrate. Also shown in Fig. 4 , using a plain line, are colony increments corresponding to a territory model that includes a realistic distribution, based on the Poisson distribution, for $L$. monocytogenes-Lac. lactis subsp. lactis separations. This calculation is performed by replacing the single inhibition time, $t^{\mathrm{N}}$, by the appropriate distribution of times and computing the population size (and colony increment) at incubation by integrating over this distribution. In general the statistical variations lead to larger colony increments corresponding to less inhibition.

Data points from Fig. 3 superimposed onto Fig. 4, clearly show that there is a strong, qualitative agreement between the results from a territory model and those from 'propinquity' experiments. It should be noted that Fig. 4 does not include any effort to 'fit' the inhibited growth of $L$. monocytogenes colonies. In fact Fig. 4 indicates that $n_{\infty}=10^{9}$ is an overestimate of the growth yield and points to a role for cell death, indicated by colony increments $<1$, in improved modelling. It is 
equally apparent that the simplified model presented above omits important details such as bacterial lag times, asymmetric growth kinetics, specifics of the inhibition response, loss of viability and the microscopics of inhibitor transport. Such refinements can readily be incorporated into the full integral form of the territory model and some of these will be explored further. We also note that the incubation time may act as a variable and therefore a full representation of the territory model would expand on Fig. 4 to give a threedimensional picture of spatio-temporal effects in interacting bacterial populations. More significantly, however, inspection of this limited form of the model already points to an important effect that should arise from correlations between statistical variations of the territory volumes and the relative colony positions. Thus, in a culture for which the territory volumes are not all equal, the large territories will be preferentially associated with colonies that are further from neighbouring colonies and therefore further from inhibitor sources. The territory model indicates that positive correlations of this form are expected to push the colony increments upwards, i.e. towards larger increments. This implies that statistical details of colony location have an important role during the development of immobilized bacterial populations and, potentially, this feature may be used to promote microbial growth responses such as strong inhibition, in complex environments such as processed foods.

\section{DISCUSSION}

$\mathrm{LAB}$ are used as starter cultures within certain fermented foods not only to enhance the flavour but also to inhibit the growth of undesirable micro-organisms. Physicochemical conditions in food favour the growth of LAB. In fermented sausages, for example, the combined effects of added salt, reduced redox potential and product drying enable lactobacilli to successfully compete against the many other microbial species that may be present in the raw sausage mix. Katsaras \& Leistner $(1988,1991)$ reported lactobacilli growing as submerged 'nests' within the sausage matrix. Mature nests usually appeared to be pure cultures of lactobacilli. The distance between the lactobacilli nests varied from 100 to $5000 \mu \mathrm{m}$. They recommended that raw sausage batter should be thoroughly homogenized to ensure an even distribution of these nests within the sausage. This would result in the shortest mean separation distance between the nests. In the present study we used a simple model system to demonstrate this principle - that the distance separating colonies immobilized within a solid system will affect the outcome of potential interactions between them. Using this system we demonstrated that the growth of a food-borne pathogen (L. monocytogenes) within a solid medium was completely inhibited by a nisin-producing strain of Lac. lactis subsp. lactis when colonies were separated by a mean distance no greater than about $450 \mu \mathrm{m}$. Increasing the separation distance reduced the inhibitive effect. At a mean colony separation distance of $1200 \mu \mathrm{m}$ between the two species, the L. monocytogenes colonies were able to grow, with an increment of over $10^{3}$ c.f.u. per colony.

By using nisin-producing and -non-producing strains of Lac. lactis subsp. lactis and by buffering the medium which prevented an increase in acidity, the effects of nisin and acidity could be investigated separately. Both effects were strongly inhibitive. When the effects of nisin and acidity were eliminated from the mixed culture (by buffering and by using the nisin-non-producing Lac. lactis subsp. lactis MG1614) L. monocytogenes, at a separation distance of $500 \mu \mathrm{m}$, showed a colony growth increment of $5 \times 10^{4}$ c.f.u. per colony (Fig. 3), a log reduction of only 0.1 compared to the control. This demonstrated that inhibition due to other possible interactive effects (such as depletion of substrate, oxygen starvation, hydrogen peroxide production, etc.) was not significant compared to the inhibitive effects of nisin (Fig. 3) and acidity (Fig. 2). At $500 \mu \mathrm{m}$ and in the presence of nisin-producing Lac. lactis subsp. lactis FI5876, L. monocytogenes showed a colony growth increment of 3.9 c.f.u. per colony in unbuffered medium, a $\log$ reduction of 3.5 from the pure culture (Fig. 1) and $5 \cdot 0$ c.f.u. per colony in buffered medium, a log reduction of $4 \cdot 1$ (Fig. 3). At a separation of $500 \mu \mathrm{m}$ in the presence of the nisin-non-producing strain Lac. lactis subsp. lactis MG1614 but in unbuffered medium, L. monocytogenes showed a colony growth increment of 10 c.f.u. per colony, a log reduction of $3 \cdot 1$ (Fig. 2). These data suggest that acidity had a greater inhibitive effect than nisin.

Food is often of solid or semi-solid consistency and is usually spatially heterogeneous. The growth of microorganisms, whether they are starter cultures or undesired contaminants, within a solid food product increases the heterogeneity of the food. Colonies may produce gradients of $\mathrm{pH}$, substrates, oxygen and bacteriocins, etc. For example, Mitchell (1995) measured steep $\mathrm{pH}$ gradients surrounding a colony of Enterococcus faecalis growing in a medium containing $0.5 \%$ $(\mathrm{w} / \mathrm{v})$ glucose (about $\mathrm{pH} 7)$. At the location of the colony the $\mathrm{pH}$ of the medium fell to 4.96 after $48 \mathrm{~h}$ incubation at $30^{\circ} \mathrm{C}$, whereas at a distance of $1500 \mu \mathrm{m}$ from the colony centre the medium was at $\mathrm{pH} 6.7$. Similarly the $\mathrm{pH}$ of the medium in the locality of a Staphylococcus aureus colony grown under similar conditions was about $\mathrm{pH} 5 \cdot 5$, but at a distance of $1700 \mu \mathrm{m}$ from the colony the $\mathrm{pH}$ was $6 \cdot 7$. Comparison of the colony growth increment of the strains in pure culture in the present study illustrates the effect of these acid gradients in restricting colony growth both in mixed and in pure cultures (the latter contributing to the 'overcrowding effect'). For instance, at about $300 \mu \mathrm{m}$ separation distance, L. monocytogenes in pure culture (Figs 1 and 2) showed a colony growth increment of $1.9 \times 10^{4}$ c.f.u. per colony in buffered medium (i.e. with added $\mathrm{CaCO}_{3}$ ), but in unbuffered medium at this separation distance the colony growth increment was only $6 \cdot 3 \times 10^{2}$ c.f.u. per colony (Fig. 3 ). In comparison, at the same separation distance, the nisin-producing Lac. lactis subsp. lactis FI5876 colony growth increment in buffered medium was 3.6 c.f.u. per colony and in 
unbuffered medium was $3 \cdot 3$. Thus, Lac. lactis subsp. lactis, as would be expected, was less affected by acidity, although the L. monocytogenes colony growth increment was greater that of Lac. lactis subsp. lactis in buffered medium.

The distance within which colonies are able to interact (the zone of influence) will vary according to a range of factors: e.g. the particular organisms, their relative numbers, specific growth rate, growth lag, motility, generation of inhibitor, nutrient type and concentration, diffusion coefficients of substrates and inhibitors, affinity constants for substrates, inhibition constants for inhibitors, abiotic variables and time. The method described in this paper allows the measurement of the relationship between interactive response and colony separation distance. The mathematical description of this relationship, incorporating some of the important variables and details of the statistical geometry, is developed in terms of a simplified territory model. This model, which will be developed further, gives a clear picture of the role of structure and transport in the growth of immobilized bacterial populations. In particular this model is able to highlight correlations between relative colony positions and bacterial growth responses that, potentially, are important in the development and control of mixed populations.

\section{ACKNOWLEDGEMENTS}

This work was supported by contract FG72/539 from BBSRC. G. C. Barker was supported by the Ministry of Agriculture, Fisheries and Food, UK. We thank IFR Norwich and Campden Food and Drink Research Association for the bacterial strains. We thank Aplin \& Barrett for the gift of nisin.

\section{REFERENCES}

Adams, M. R. \& Moss, M. O. (editors) (1995). Food Microbiology. Cambridge, UK: The Royal Society of Cambridge.

Costilow, R. N. (1981). Biophysical factors in growth. In Manual of Methods for General Microbiology, p. 69. Edited by P. Gerhardt, R. G. E. Murray, R. N. Costilow, E. W. Nester, W. A. Wood, N. R. Krieg \& G. B. Phillips. Washington, DC: American Society for Microbiology.

Delves-Broughton, J. (1990). Nisin and its uses as a food preservative. Food Technol 44, 100-117.

Hurst, A. (1981). Nisin. Adv Appl Microbiol 27, 85-123.

Jones, D., Pell, P. A. \& Sneath, P. H. A. (1984). In Maintenance of Microorganisms, pp. 35-40. Edited by B. E. Kirsop \& J. J. S. Sneath. London: Academic Press.

Katsaras, K. \& Leistner, L. (1988). Topographie der Bakterien in der Rohwurst. Fleischwirtschaft 68, 1295-1298.

Katsaras, K. \& Leistner, L. (1991). Distribution and development of bacterial colonies in fermented sausages. Biofouling 5, 115-124.

Mitchell, A. J. (1995). Modelling microbial colony growth in a solid phase food system. PhD Thesis, University of Wales Cardiff.

Schillinger, U., Geisen, R. \& Holzapfel, W. H. (1996). Potential of antagonist microorganisms and bacteriocins for the biological preservation of foods. Trends Food Sci Technol 7, 158-164.

Vandenbergh, P. A. (1993). Lactic acid bacteria, their metabolic products and interference with microbial growth. FEMS Microbiol Rev 12, 221-238.

Wimpenny, J. W. T., Leistner, L., Thomas, L. V., Mitchell, A. J., Katsaras, K. \& Peetz, P. (1995). Submerged bacterial colonies within food and model systems: their growth, distribution and interactions. Int J Food Microbiol 28, 299-315.

Received 18 December 1996; revised 10 March 1997; accepted 25 March 1997. 